\title{
Identification of the ferroptosis-related long non-coding RNAs signature to improve the prognosis prediction and immunotherapy response in patients with NSCLC
}

\author{
Meng $\mathrm{Li}^{1,2 \dagger}$, Yanpeng Zhang ${ }^{3,2,4 \dagger}$, Meng Fan ${ }^{1 \dagger}$, Hui Ren ${ }^{1,2}$, Mingwei Chen ${ }^{1 *}$ and Puyu Shi ${ }^{{ }^{*}}$
}

\begin{abstract}
Background: Non-small cell lung cancer (NSCLC) is the most prevalent type of lung carcinoma with an unfavorable prognosis. Ferroptosis is involved in the development of multiple cancers. Whereas, the prognostic value of ferroptosis-related IncRNAs in NSCLC remains uncertain.

Methods: Gene expression profiles and clinical information of NSCLC were retrieved from the TCGA database. Ferroptosis-related genes (FRGs) were explored in the FerrDb database and previous studies, ferroptosis-related IncRNAs (FRGs-IncRNAs) were identified by the correlation analysis and the LncTarD database. The differentially expressed FRGs-IncRNAs were screened and FRGs-IncRNAs associated with the prognosis were explored by univariate Cox regression analysis and Kaplan-Meier survival analysis. Then, an FRGs-IncRNAs signature was constructed and verified by the Lasso-penalized Cox analysis. Finally, the potential correlation between risk score, immune checkpoint genes, and chemotherapeutic sensitivity was further investigated.

Results: 129 IncRNAs with a potential regulatory relationship with 59 differentially expressed FRGs were found in NSCLC, of which 10 were related to the prognosis of NSCLC $(P<0.05) .9$ prognostic-related FRGs-IncRNAs were used to construct the prognostic model and stratify NSCLC patients into high- and low-risk groups. A worse outcome was found in patients with high risk $(P<0.05)$. Moreover, a good predictive capacity of this signature in predicting NSCLC prognosis was confirmed. Additionally, 45 immune checkpoint genes and 4 chemotherapeutics drugs for NSCLC were identified to be correlated with the risk score.
\end{abstract}

Conclusion: A novel FRGs-IncRNAs signature was successfully constructed, which may contribute to improving the management strategies of NSCLC.

Keywords: Non-small cell lung cancer, Ferroptosis, Long non-coding RNAs, Prognostic, Signature

*Correspondence: chenmw36@163.com; chenmingwei@mail.xjtu.edu.cn; carrot.0424@163.com; shipuyu@xjtufh.edu.cn

${ }^{\dagger}$ Meng Li, Yanpeng Zhang and Meng Fan have contributed equally to this work and share first authorship

${ }^{1}$ Department of Respiratory and Critical Care Medicine, The First

Affiliated Hospital of Xi'an Jiao Tong University, No. 277, Yanta West Road,

Xi'an 710061, Shaanxi, China

Full list of author information is available at the end of the article

\section{Introduction}

Lung cancer ranks top in the incidence of malignancies and imposes an enormous socio-economic burden worldwide [1]. Non-small-cell lung cancer (NSCLC) is the most prevalent subtype of primary lung cancer, among which adenocarcinoma (LUAD) as well as squamous cell carcinoma (LUSC) is the leading histological type [2,3]. Although there have been breakthroughs 
in the targeted therapy and immunotherapy of lung cancer, the long-time survival rate of NSCLC remains unsatisfactory [4-6], and most patients will inevitably develop drug resistance. Since most anti-tumor drugs play a therapeutic role by inducing apoptosis of cancer cells, it is of great significance to pinpoint novel cell death pathways in NSCLC and discover new directions for identifying treatment strategies and evaluating prognosis.

Ferroptosis, a newly discovered mode of irondependent regulated cell death unlike apoptosis, pyroptosis, autophagy and necrosis, is mainly caused by the iron accumulation-mediated lipid peroxidation and exhibits peculiar morphology, genetics, as well as biochemistry features [7]. The development of disease and organisms and anti-tumor drug resistance has also been verified to be affected by ferroptosis $[8,9]$. Long non-coding RNAs (lncRNAs) are a kind of RNA with more than 200 bases in length. Although lncRNAs lack protein-coding ability, it is still considered a target for gene therapy of cancer since it is involved in the tumor occurrence, development as well as metastasis by modulating gene expression at chromatin, transcriptional, and post-transcriptional levels [10, 11]. Additionally, some lncRNAs were found to inhibit ferroptosis by acting as competitive endogenous RNA to prevent oxidation in various cancers including lung cancer $[12,13]$. Metallothionein 1D pseudogene, a lncRNA, was found to sensitize erastin-induced ferroptosis in NSCLC by modulating the miR-365a-3p/NRF2 axis [14]. A G3BP1-interacting lncRNA was demonstrated to promote ferroptosis via nuclear sequestration of p53 in lung carcinoma [15]. Recently, several studies have constructed some prognostic signatures in many cancers, including LUAD [16], head and neck squamous cell carcinoma [17], bladder [18], and colon cancer [19], by exploring the prognostic ferroptosis-related lncRNAs to assist clinicians to evaluate the prognosis of patients. Whereas, the signature of ferroptosis-related lncRNAs and its association with prognosis in NSCLC has not been systematically evaluated.

Herein, we explored the expression of ferroptosisrelated genes (FRGs) and ferroptosis-related lncRNAs (FRGs-lncRNAs) in NSCLC and further investigate the relationship between these lncRNAs and the prognosis in NSCLC based on the Cancer Genome Atlas (TCGA) database. Subsequently, a prognostic model was constructed on the basis of FRGs-lncRNAs in the training set and verified by internal and external validation. Furthermore, the relationship between risk score, clinicopathological features, immune checkpoint genes (ICGs), and chemotherapeutics sensitivity was further elucidated in NSCLC.

\section{Materials and methods}

\section{Data acquisition and processing}

Gene expression profiles and clinical features of TCGALUAD (510 tumors and 58 normal) and TCGA-LUSC (496 tumors and 49 normal) were obtained from UCSC Xena (https://xena.ucsc.edu/). The expression profiles of LUAD and LUSC were combined as NSCLC expression matrix, and a total of 1093 patients (986 tumors and 107 normal) were eventually included in the present study after the batch correction by using ComBat function of the R "sva" package. No ethics committee approval and informed consent of patients were required in this study since the data were obtained from a public database.

\section{Identification of FRGs}

259 FRGs (Additional file 2: Table S1) were explored from the "Marker", "Driver" and "Suppressor" modules of the FerrDb database (http://www.zhounan.org/ferrdb/) [20] and previous studies [8, 21-23], among which 241 were found to be expressed in NSCLC on the basis of available mRNA expression data. The expression profiles of these FRGs were extracted to identify the differentially expressed FRGs (DE-FRGs) between normal and tumor by the "limma" package of R. $|\log 2 \mathrm{FC}|>1$ and $\mathrm{FDR}<0.05$ is considered to be significant.

\section{Functional enrichment analysis}

"ClusterProfiler" of $\mathrm{R}$ was utilized to conduct Gene Ontology (GO) as well as Kyoto Encyclopedia of Genes and Genomes (KEGG) analysis to investigate the biological functions and signaling pathways affected by these DE-FRGs [24-26].

\section{Screening of FRGs-IncRNAs}

Pearson correlation analysis was conducted to identify the potential lncRNAs correlated with DE-FRGs based on the TCGA database. $|\mathrm{R}|>0.5$ and $P<0.001$ were considered a strong correlation. In addition, the lncRNAs that have a regulatory relationship with DE-FRGs and expressed in NSCLC were further explored and screened in the LncTarD database (http://bio-bigdata.hrbmu.edu. cn/LncTarD/) [27]. Then, IncRNAs that have an expression and regulatory relationship with DE-FRGs in TCGA and LncTarD database were unionized to obtain candidate FRGs-lncRNAs. The "survival" package, univariate Cox regression analysis, and Kaplan-Meier (K-M) survival method were conducted to investigate FRGs-lncRNAs associated with the prognosis in NSCLC.

\section{FRGs-IncRNAs prognostic model construction}

986 NSCLC patients were randomly separated into training and verification set at a ratio of 7:3 by R. Lasso-penalized Cox regression analysis was performed to establish 
an FRGs-lncRNAs prognostic model in the training set using the $\mathrm{R}$ "glmnet" package $[28,29]$. The risk score of each sample was calculated on the basis of the normalized expression levels and the corresponding Lasso's coefficient of the FRGs-lncRNAs [Risk score $=\sum \exp (\mathrm{i}){ }^{*} \operatorname{coef}(\mathrm{i})$ ], and patients were further categorized as high- and lowrisk groups in accordance with the median risk score. Then "ggplot2" package of $\mathrm{R}$ was performed to draw the survival scatter plot. K-M survival curve and receiver operational characteristic (ROC) curves were generated to evaluate the relationship between risk score and prognosis, and the predictive capacity of the signature. Additionally, the expression levels of FRGs-lncRNAs of each sample in the two risk groups were uncovered by the "heatmap" package. Finally, the results were further identified in the internal and external validation groups.

\section{Predictive nomogram construction}

Wilcoxon test was performed to investigate the potential relationship between risk score and multiple clinical features (EGFR mutation, ALK-EML4 rearrangement, age, gender, stage, and TNM stage). $P<0.05$ was considered to be significant. Subsequently, the independent prognostic factors were investigated by univariate and multivariate Cox regression analysis and visualized the results by $\mathrm{R}$ "forestplot" package. Finally, a nomogram was established integrating the independent risk factors $(\mathrm{T}, \mathrm{N}$ and Risk score) and $\mathrm{M}$ stage with important clinical prognostic significance for predicting 1,3 , and 5 -year overall survival (OS) of NSCLC patients by using the "rms" package of $R$, and the prediction accuracy of which was further evaluated by the calibration and ROC curve.

\section{Correlation analysis between risk score, ICGs and chemotherapeutic drugs sensitivity}

79 ICGs were explored from the previous literature [30], of which 66 were expressed in NSCLC. Student t-test was used for difference analysis, and the top 10 ICGs with significant differences were shown as boxplots. The correlation between risk score and sensitivity of chemotherapeutics commonly used for NSCLC treatment, including cisplatin, etoposide, docetaxel, gefitinib, erlotinib, gemcitabine, paclitaxel, was evaluated by using Wilcoxon signed-rank test of the $\mathrm{R}$ package "pRRophetic" and "ggplot2". $P<0.05$ is considered a significant correlation.

\section{Statistical analysis}

Perl and R (4.0.1) were utilized for data processing and statistical analysis. Benjamini-Hochberg method was performed to identify the DE-FRGs. Pearson correlation analysis were conducted to explore the lncRNAs associated with DE-FRGs. FRGs-lncRNAs associated with prognosis were explored by univariate Cox regression tests and K-M survival analysis in NSCLC. An FRGslncRNAs prognostic model was generated by the Lassopenalized Cox regression analysis and further evaluated the predictive capacity by K-M survival and ROC curve analysis. Independent predictors of OS in NSCLC were identified by multivariate Cox regression analysis. $P<0.05$ was considered significant.

\section{Results}

Identification and enrichment analysis of FRGs in NSCLC 986 patients with NSCLC and 107 healthy control from the TCGA database were enrolled in the present study. 259 FRGs were explored from the FerrDb database and previous studies, among which 241 were found to be expressed in NSCLC (Additional file 2: Table S1). The process of our research is shown in Fig. 1. Herein, we found 59 FRGs were significantly differentially expressed between normal and NSCLC patients (28 downregulated and 31 upregulated, Fig. 2A, B). The DE-FRGs were determined mainly involved in biological pathways related to the response to oxidative stress, lipid droplet, and oxidoreductase activity biological function by $\mathrm{GO}$ analysis (Fig. 3A). KEGG enrichment analysis revealed that these DE-FRGs also participate in ferroptosis, arachidonic acid metabolism, glutathione metabolism, and NOD-like receptor signaling pathway (Fig. 3B).

\section{Identification of the ferroptosis-related IncRNAs in NSCLC}

104 FRGs-lncRNAs were identified by Pearson correlation analysis $(|\mathrm{R}|>0.5, P<0.001)$ and the top 10 of which were shown in Fig. 4A. Next, 44 lncRNAs that have a regulatory relationship with DE-FRGs were explored in the LncTarD database, and 30 of them were determined to be expressed in NSCLC. The interaction network between DE-FRGs and IncRNAs in the LncTarD database were shown in Fig. 4B. Finally, 129 candidate FRGslncRNAs were collected in this study by unifying the two methods (Fig. 4C, Additional file 2: Table S2). Then, 13 FRGs-lncRNAs associated with NSCLC prognosis were identified by univariate Cox analysis (Fig. 5A, Additional file 2: Table S3), among which, 10 lncRNAs also showed significant results in K-M analysis and were selected for the prognostic model construction (Additional file 2: Table S4). The survival curves of the first 6 lncRNAs were demonstrated in Fig. 5B-G and the emperical cumulative density function (ecdf) plots of all 10 lncRNAs were shown in Additional file 1: Figure S1 $(P<0.05)$.

\section{Construction of the prognostic model based on ferroptosis-related IncRNAs}

986 NSCLC patients were categorized into training and testing cohorts randomly at a ratio of 7:3, and 9 of the 


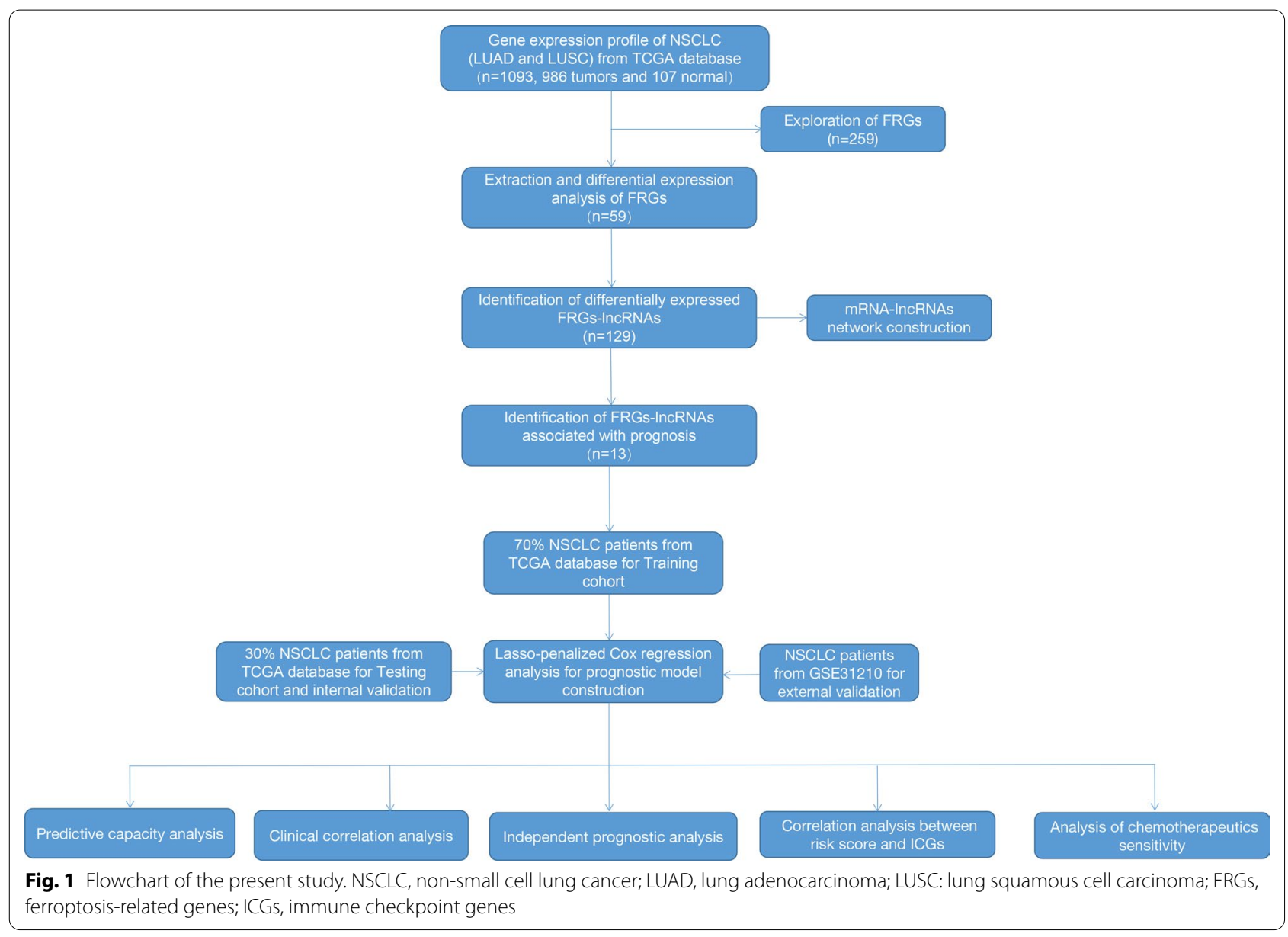

10 prognostic FRGs-lncRNAs (AQP4-AS1, DANCR, LINC00460, LINC00892, LINC00996, MED4-AS1, SNHG7, UCA1, and WWC2-AS2) were eventually used for prognostic signature construction (Fig. 6A, B, Additional file 2: Table S5). Then, patients were classified into a high and low-risk group in accordance with the median of risk score [Risk score $=\exp (\mathrm{AQP} 4-\mathrm{AS} 1)$ * $-0.268+\exp ($ DANCR $) *-0.041+\exp ($ LINC00460)

* $0.122+\exp ($ LINC00892) * $-0.390+\exp ($ LINC00996)

* $-0.144+\exp ($ MED4-AS1) $*-0.198+\exp ($ SNHG7)

$*-0.028+\exp (\mathrm{UCA} 1) * 0.092+\exp (\mathrm{WWC} 2-\mathrm{AS} 2) *$

0.497 ] and the proportion of dead patients is higher in the patients with high-risk score (Fig. 6C, D). Additionally, the K-M plot and ROC curve analysis illustrated a worse prognosis in the NSCLC patients with the highrisk and a good predictive capacity of the signature, respectively $(P<0.01$, AUC $=0.606$, Fig. $6 \mathrm{E}, \mathrm{F})$. The expression levels of the 9 FRGs-lncRNAs between the two risk groups are presented in Fig. 6G and Additional file 1: Figure S2.

\section{Validation of the prognostic signature}

To evaluate the robustness of the prognostic model, the patients from the testing cohort were also grouped according to the median of the same risk score (Fig. 7A), and the survival status scatter plot, K-M plot and ROC curve analysis demonstrated the similar results to the training group $(P<0.01, \mathrm{AUC}=0.604$, Fig. $7 \mathrm{~B}-\mathrm{D})$. The expression trend of the 9 FRGs-LncRNAs between the two risk groups of the testing cohort was similar to that of the training group (Fig. 7E, Additional file 1: Figure S3).

In addition, external validation was also carried out in GES31210 to further verify the effectiveness of this prognostic model. Here, 8 lncRNAs (without MED4-AS1) were utilized for validation since MED4-AS1 expression was not found in NSCLC in this cohort. The results of the survival status scatter plot and heatmap in this cohort were similar to those of the training group (Additional file 1: Figure S4A-B, Figure S4E). and ROC curve and $\mathrm{K}-\mathrm{M}$ plot analysis also illustrated that the good prediction performance of this prognostic signature and the correlation between the risk score based on these 8 lncRNAs 

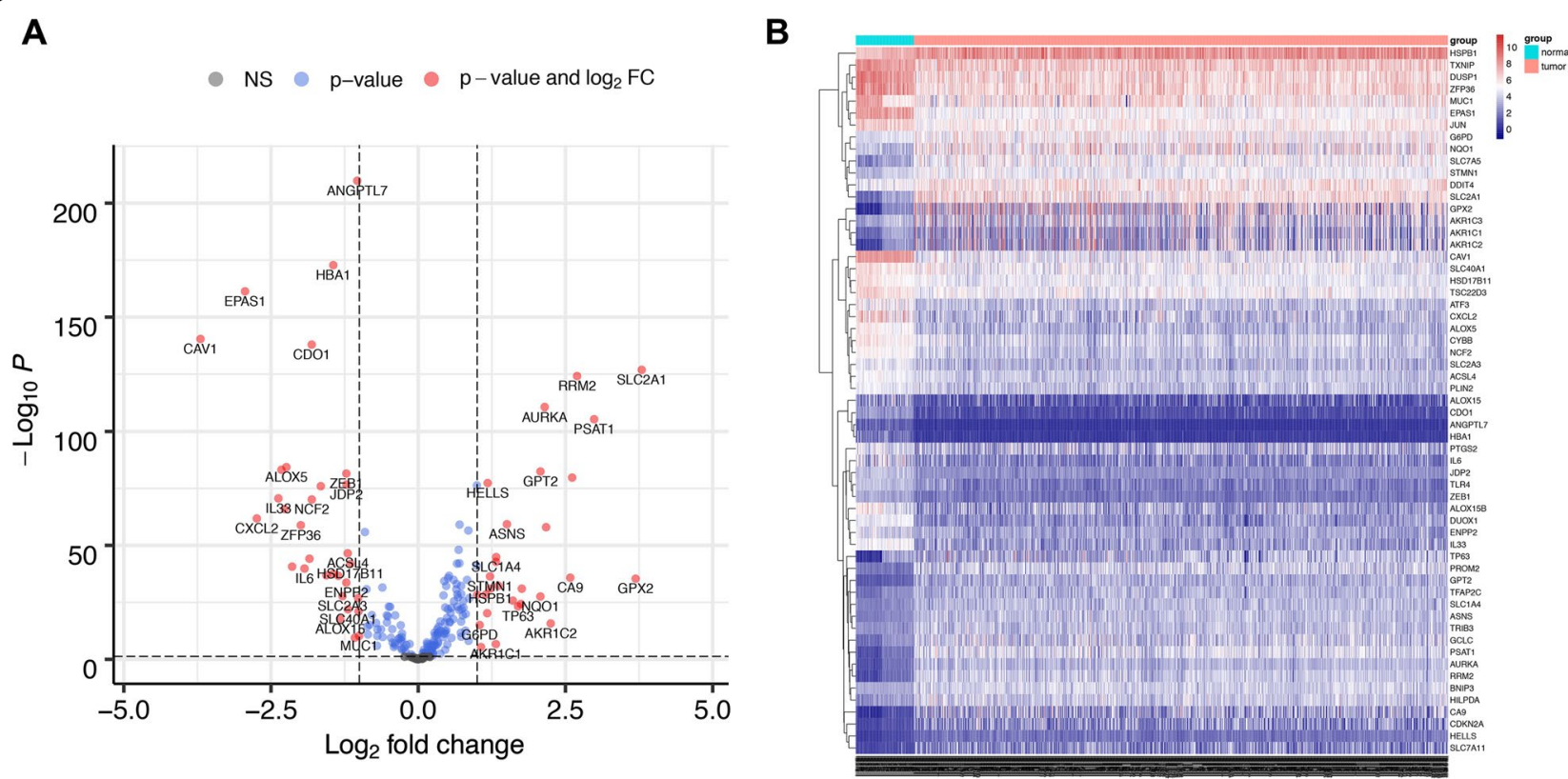

Fig. 2 Identification of the FRGs in patients with NSCLC. The DE-FRGs in NSCLC were shown in the volcano plot (A) and heatmap (B). FDR $<0.05$ and $|\log 2 \mathrm{FC}| \geq 1$ were considered significant. The gray dots in $\mathbf{A}$ represent the FRGs with no statistical difference, the blue dots represent the FRGs with $F D R<0.05$, and the red dots represent genes with $F D R<0.05$ and $|\log 2 F C| \geq 1$. FRGs, ferroptosis-related genes; DE-FRGs, differentially expressed FRGs; NSCLC, non-small cell lung cancer; FDR, false discovery rate

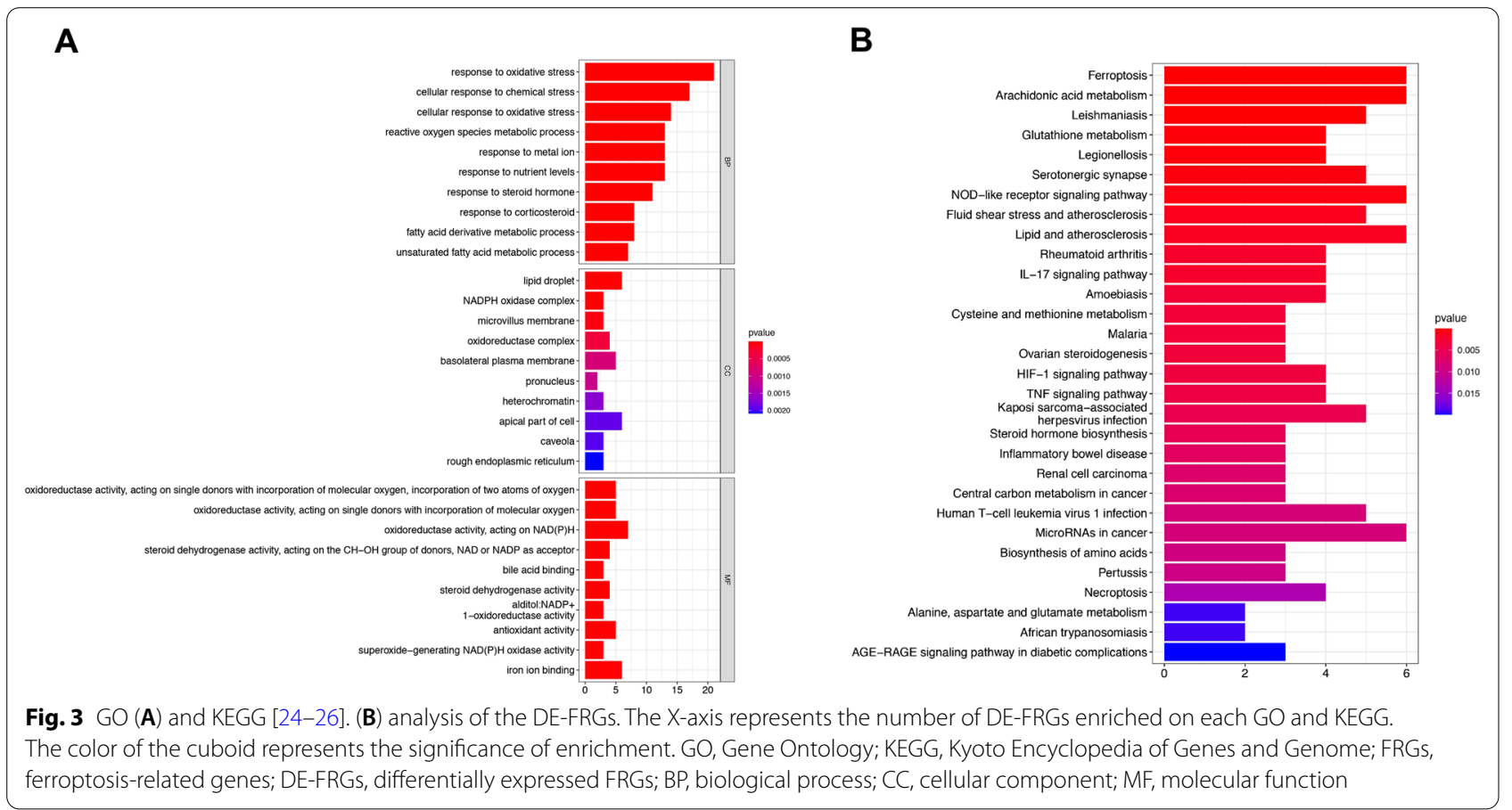



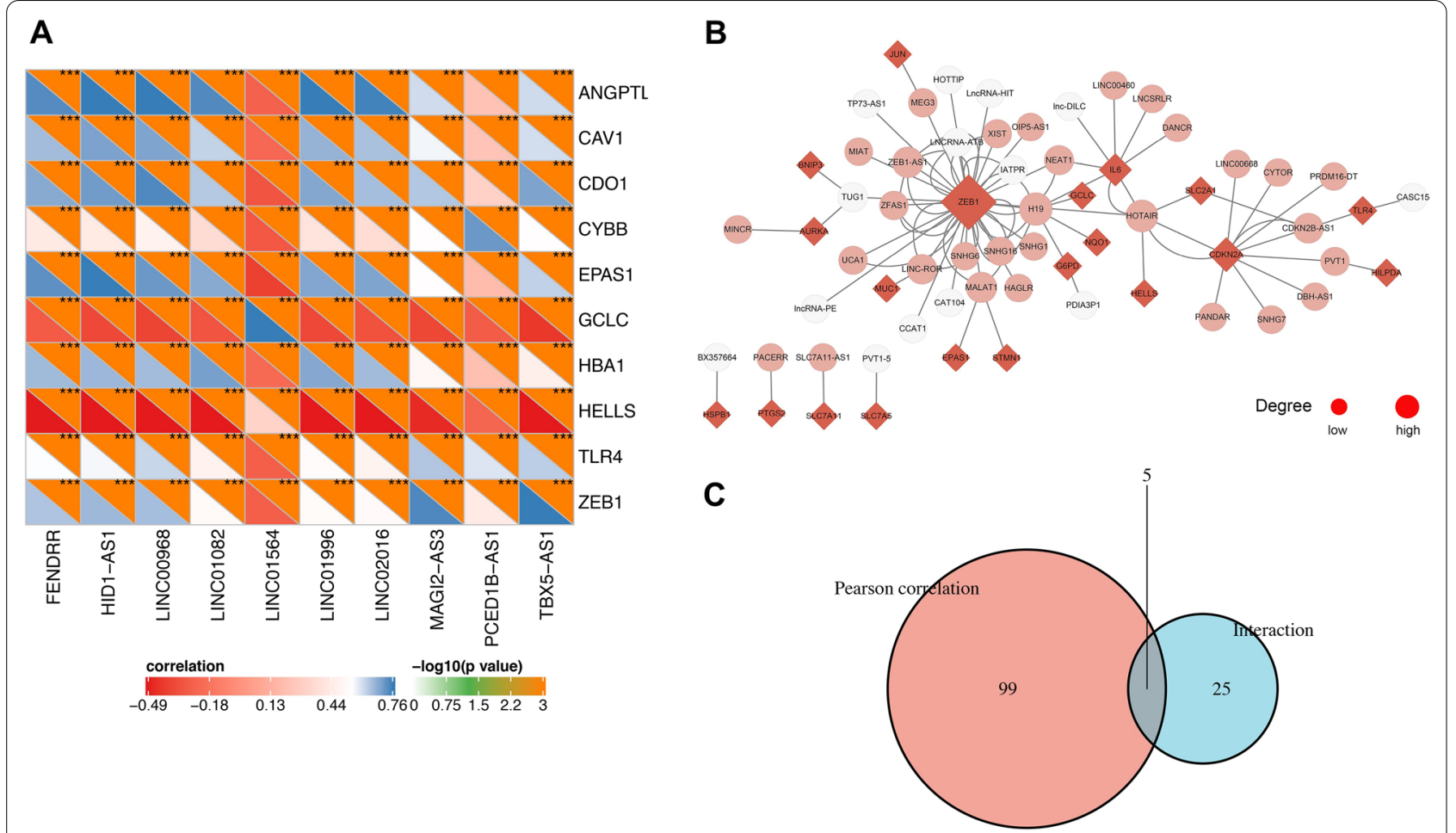

Fig. 4 Identification of the FRGs-IncRNAs in NSCLC. (A) Correlation heatmap of the top 10 FRGs and IncRNAs based on TCGA database. The criteria of correlation analysis: $|R|>0.5$ and $P<0.001$. (B) Interaction network between DE-FRGs and IncRNAs in LncTarD database. The DE-FRGs and IncRNAs are shown as the red diamond and pink circle, respectively. The white circles represent IncRNAs that are not expressed in NSCLC. The size of the dot is positively correlated with the degree of correlation. (C) The union set of the DE-FRGs related IncRNAs in the TCGA database and LncTarD database were shown as a Venn diagram. FRGs, ferroptosis-related genes; IncRNAs: long non-coding RNAs; FRGs-IncRNAs, FRGs-related IncRNAs; TCGA: the Cancer Genome Atlas; NSCLC, non-small cell lung cancer; DE-FRGs: differentially expressed FRGs

and the prognosis of NSCLC $(P=0.016$, AUC $=0.616$ Additional file 1: Figure S4C and D).

\section{Correlation analysis between risk score and clinical characteristics and the construction of nomogram}

As shown in Fig. 8A-H, the results illustrated that risk score was related to gender, stage and $\mathrm{T}, \mathrm{N}$ stage, among which, male patients and those with higher $\mathrm{T}$ and $\mathrm{N}$ stage had higher risk. Further subgroup analysis found that the $\mathrm{T}$ stage is correlated with risk score in both LUAD and LUSC, and stages and N stage were found to relate to the risk score in LUAD, and $M$ stage showed a relationship with the risk score in LUSC (Additional file 1: Figure S5).

Univariate and multivariate Cox regression analysis elucidated that risk score, $\mathrm{T}$, and $\mathrm{N}$ stages are independent risk factors for the OS of NSCLC patients $(P<0.05$, Fig. 8I, J). Then, a nomogram merging independent risk factors ( $\mathrm{T}, \mathrm{N}$ and Risk score) and $\mathrm{M}$ stage with important clinical prognostic significance were constructed (Fig. 9A), and a satisfactory agreement between the predicted and observed values at the probabilities of 1-, 3and 5-year survival was shown in the calibration curve
(Fig. 9B). What's more, the ROC curve revealed that the risk score was more accurate in predicting survival in patients with NSCLC than traditional clinical features (Fig. 9C).

\section{Correlation analysis between risk score, ICGs and chemotherapeutics sensitivity}

The results showed a substantial difference in the expression of 45 ICGs between the two risk groups (Additional file 2: Table S6), and the first 10 ICGs (BTLA, BTN2A2, CD160, CD226, CD27, CD276, CD40LG, CD96, CTLA4, TIGIT) were presented in Fig. 10A. As shown in Fig. 10B-H, the correlation analysis between the risk score and the sensitivity of chemotherapeutics for NSCLC showed that patients with high-risk scores were highly sensitive to the chemotherapeutics cisplatin, docetaxel, erlotinib, and paclitaxel (all $P<0.05$ ), while there was no significant difference in the sensitivity of the etoposide, gefitinib, and gemcitabine between the two groups $(P>0.05)$. 


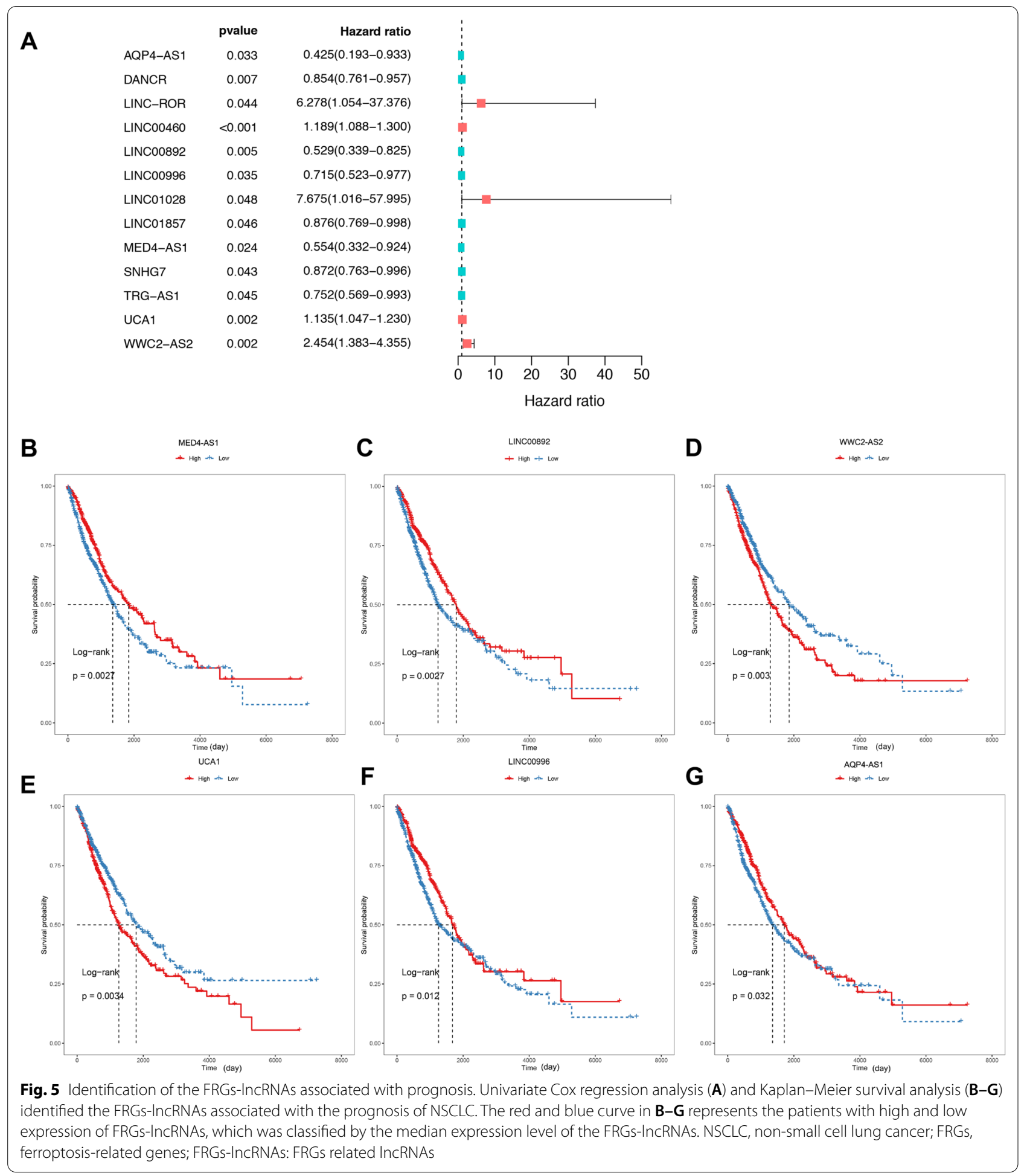

\section{Discussion}

Lung cancer, the most fatal malignancy worldwide, has a variety of histological subtypes. As the most prevalent pathological pattern of lung cancer, NSCLC patients are usually diagnosed at an advanced stage with poor survival due to the lack of early specific clinical manifestations, diagnostic and prognostic biomarkers [31]. Over the past decade, the long-term survival rate of advanced 
NSCLC has been significantly prolonged due to the development of targeted therapies and immunotherapy. Unfortunately, poor prognoses remain in some patients after systemic and targeted therapy [32,33]. Hence, there is an urgent need for safe and feasible markers that can accurately predict prognosis, so as to make the management of NSCLC patients more accurate, personalized and timely.

Ferroptosis is a newly type regulatory cell death with specific properties and recognizing functions that participated in numerous diseases including cancers [34], which was identified involved in killing malignant cells and inhibiting tumor progression in several cancers, such as NSCLC [35], pancreatic cancer [36], breast cancer [37] and hepatocellular carcinoma [38] and consequently considered as a novel therapeutic strategy for cancer treatment. Interestingly, the crucial role of IncRNAs in the regulation of ferroptosis has been increasingly recognized [39]. Whereas, the role of FRGs-lncRNAs in prognostic, immune response, and chemotherapeutic effect in NSCLC remains unclear.

Herein, we found 59 FRGs and 129 FRGs-lncRNAs differentially expressed between NSCLC and normal patients. Then, a prognostic model was established in the training set based on the 9 prognostic FRGs-lncRNAs and verified in the internal and external cohort. Additionally, the relationship between risk score and clinical characteristics of NSCLC were assessed and a nomogram was constructed. Finally, the correlation between ICGs, chemotherapeutic sensitivity as well as risk score was investigated to evaluate the potential role of FRGslncRNAs in the immune response and chemotherapeutic effect in NSCLC. These findings strongly suggest a potentially important role of FRGs-lncRNAs in NSCLC.

In our study, the predictive model was established based on the 9 FRGs-lncRNAs (DANCR, LINC00460, LINC00892, LINC00996, UCA1, and WWC2-AS2, AQP4-AS1, MED4-AS1, SNHG7,). Aquaporin 4 antisense RNA 1 (AQP4-AS1) transcribes a lncRNA with unknown function, which was found related to the risk of gastric [40] and breast cancer [41]. We found that AQP4AS1 acts as a protector factor in NSCLC. Whereas, the biological function of AQP4-AS1 in ferroptosis and lung cancer has not been systematically analyzed, which needs to be further studied. LINC00460 was also demonstrated overexpressed in NSCLC and promotes epithelial-mesenchymal transition and cell migration [42], what's more, as an FRGs-lncRNA, LINC00460 was identified as a predictor and potential therapeutic target for EGFR-TKI resistance in NSCLC [43]. Although similar results with the previous studies were found in our study, whether ferroptosis involved in the drug resistance and development of NSCLC needs to be investigated in-depth. Despite several kinds of research that have illuminated the role of LINC00892 and LINC00996 in multiple cancers, their function in NSCLC has not been studied and deserves further investigation considering its significant prognostic value in NSCLC. Urothelial carcinoma-associated 1 (UCA1) was found to promote gefitinib-resistance in NSCLC [44], knockdown of UCA1 can impair cell proliferation and promoted the gefitinib-induced cell apoptosis, which was considered as a promising therapeutic target for the NSCLC patients with EGFR ${ }^{+}$[44]. So far, no studies have been conducted on the biological function of WWC2-AS2 in NSCLC, which needs a systematic study in future. LncRNA differentiation antagonizing non-protein-coding RNA (DANCR) is known to suppress epidermal progenitor cell differentiation [45], which was identified to be overexpressed in multiple cancers, promoting malignant biological behavior cancer and chemoresistance [46]. DANCR was also found upregulated and correlated with the poor prognosis in NSCLC, and which promotes the malignancy of NSCLC thorough DANCR/ miR-138/Sox4 positive feedback loop [47]. MED4-AS1 is a novel lncRNA that is upregulated in NSCLC and is positively associated with poor differentiation and metastasis [48], The lncRNA small nucleolar RNA host gene 7 (SNHG7) was considered as an oncogenic lncRNA in NSCLC, hepatocellular carcinoma, breast cancer, and colorectal cancer $[49,50]$, which was found to modulate malignant character in LUAD through SNHG7/miRNA181/cbx7 pathway, and mediates cisplatin-resistance in NSCLC through activating PI3K/AKT pathway [51]. Whereas, despite DANCR and SNHG7 being also found up-regulated in NSCLC in our study, both of them were found as protective factors, which may stem from the different genetic backgrounds, complications of patients with varying stages of NSCLC. In addition, different from

\footnotetext{
(See figure on next page.)

Fig. 6 Construction of the FRGs-IncRNAs signature in the training group. (A) Lasso coefficients profiles of the 10 FRGs-IncRNAs. (B) Lasso regression analysis obtained 9 prognostic FRGs-InCRNAs. Distribution (C) and survival status plot (D) of NSCLC patients based on the median risk score. Kaplan-Meier survival (E) and ROC curve analysis (F) of the FRGs-IncRNAs signature in the training group. (G) Heatmap of the expression profiles of the FRGs-IncRNAs in low- and high-risk groups. Each curve in $\mathbf{A}$ represents the changing track of Lasso's coefficients of the 10 prognosis-related FRGs-IncRNAs, respectively. The Y-axis represents the Lasso's coefficient, the lower and upper $X$-axis represents $\log (\lambda)$, and the number of FRGs-IncRNAs with non-zero coefficients, respectively. In $\mathbf{B}$, the $Y$-axis represents partial likelihood deviance, the lower and upper $X$-axis represents $\log (\lambda)$ and the number of FRGs-IncRNAs corresponding to the different $\log (\lambda)$, respectively. The upper $X$-axis corresponding to the smallest partial likelihood deviance is the best number of FRGs-IncRNAs included in the model. NSCLC, non-small cell lung cancer; IncRNAs: long non-coding RNAs; FRGs, ferroptosis-related genes; FRGs-IncRNAs: FRGs related IncRNAs; ROC, receiver operating characteristic
} 

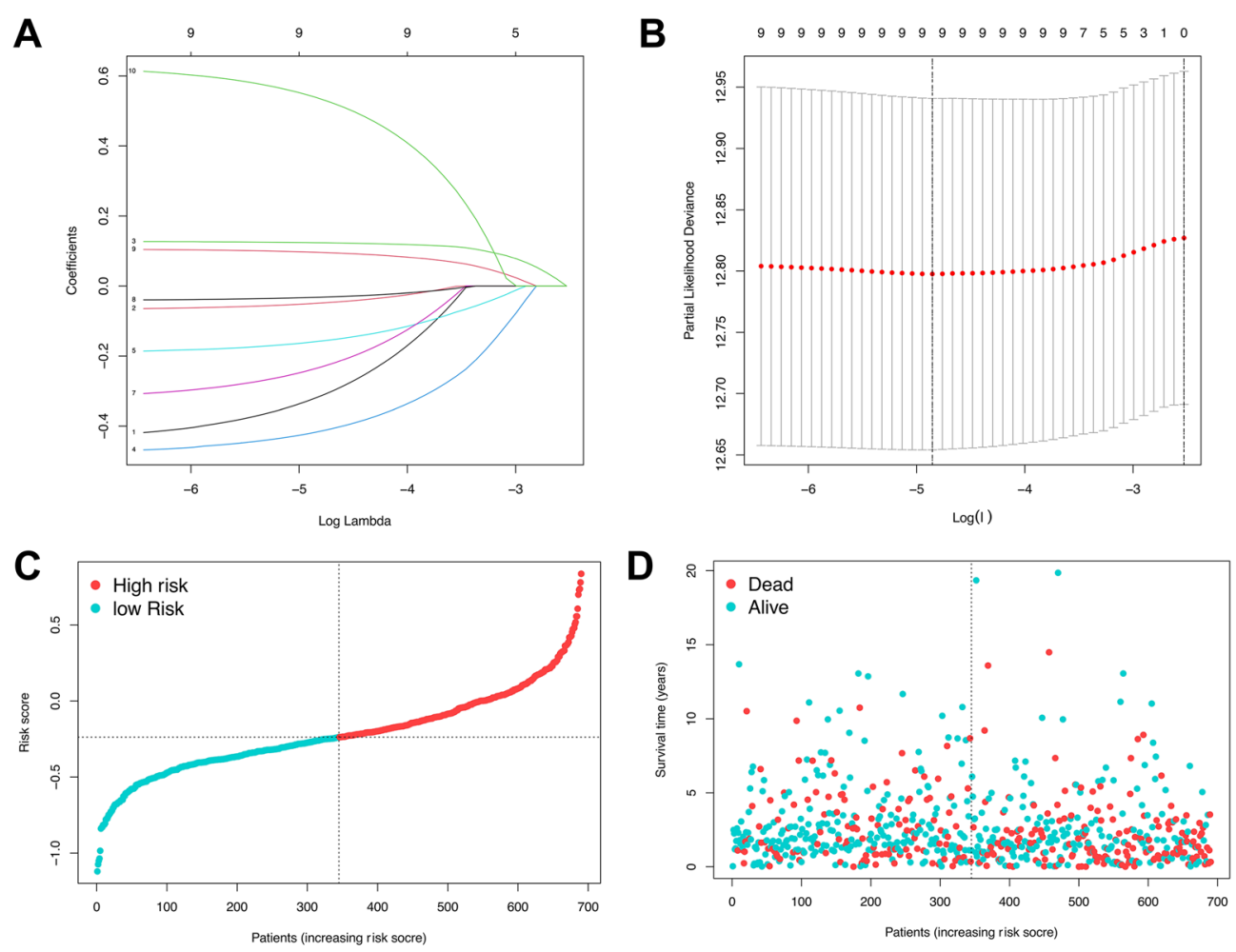

E
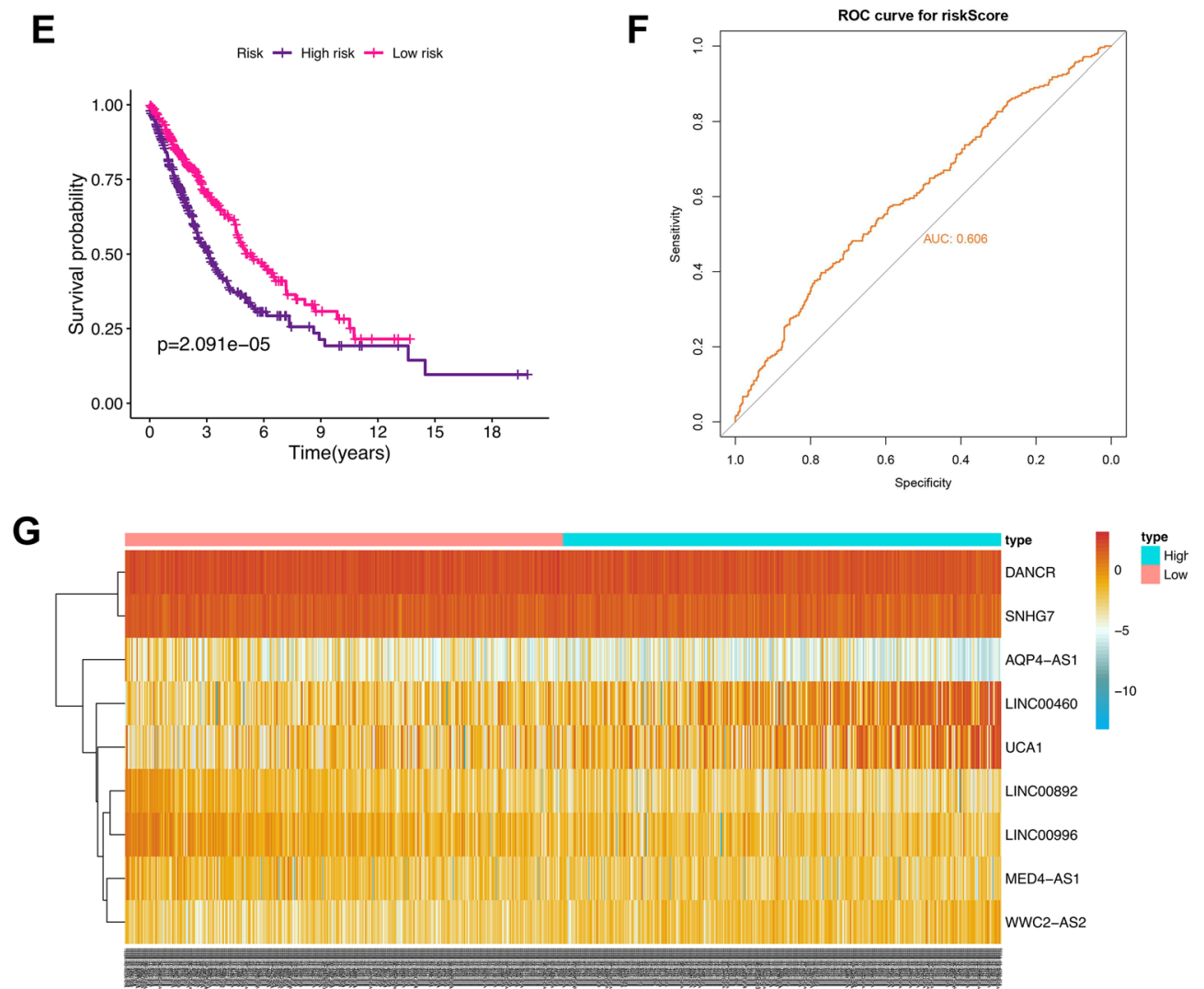

Fig. 6 (See legend on previous page.) 


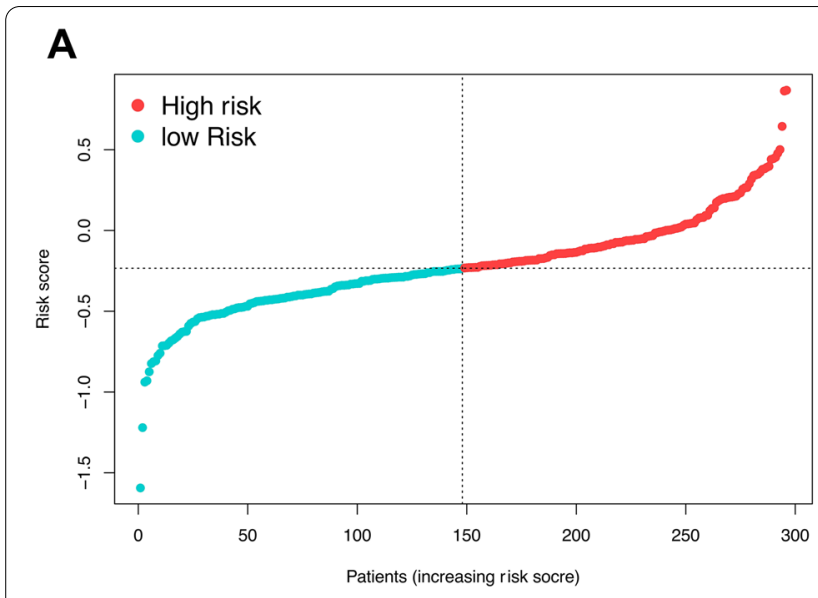

B

C
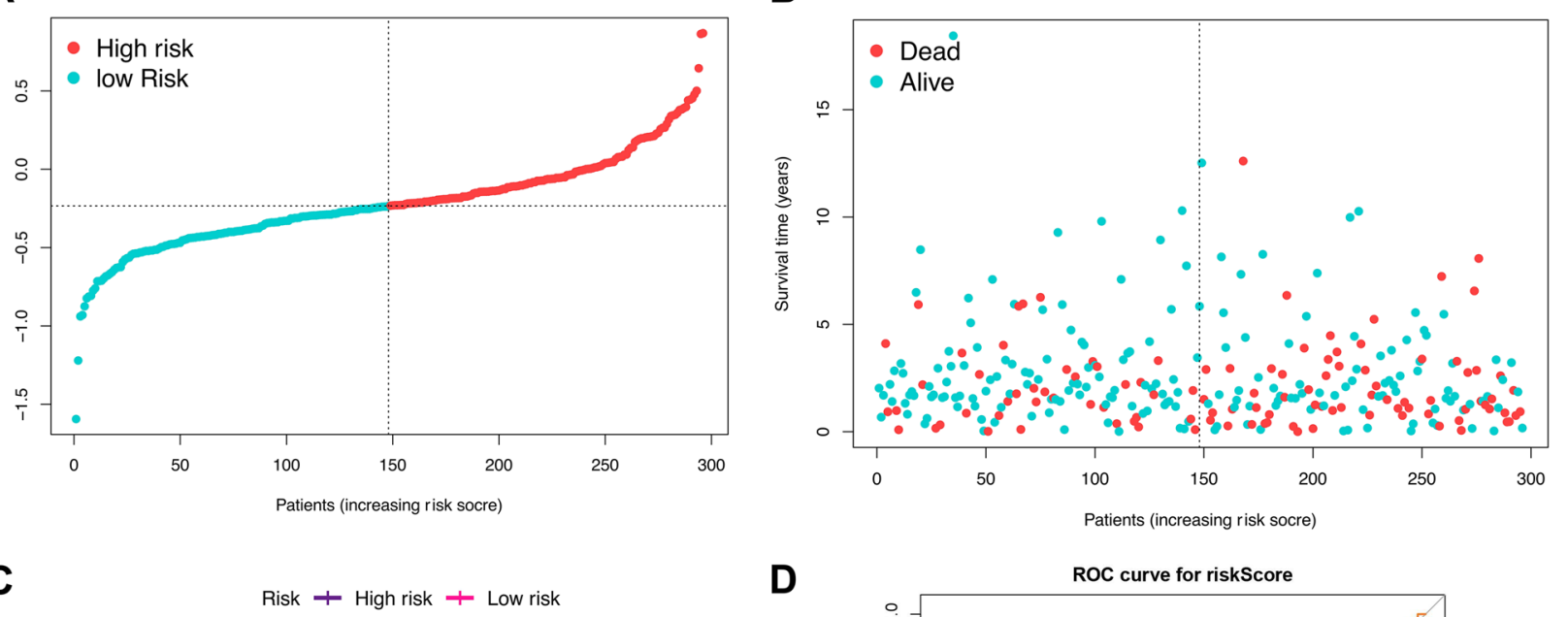

D

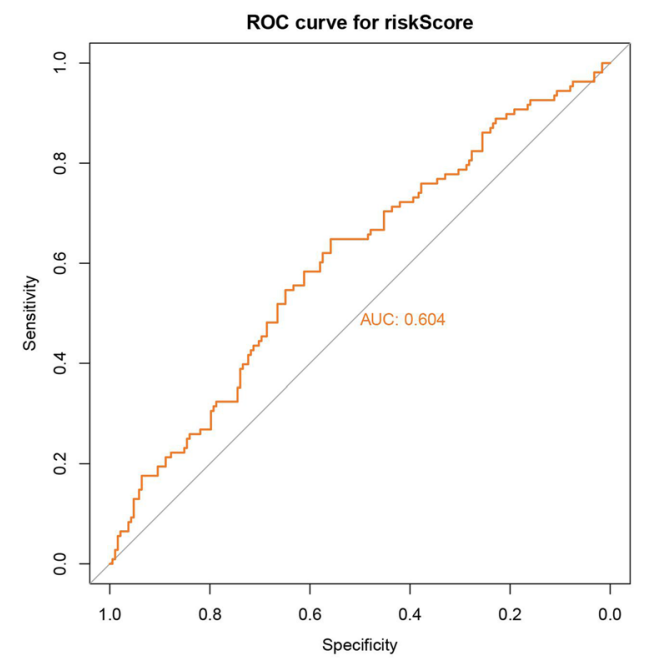

E
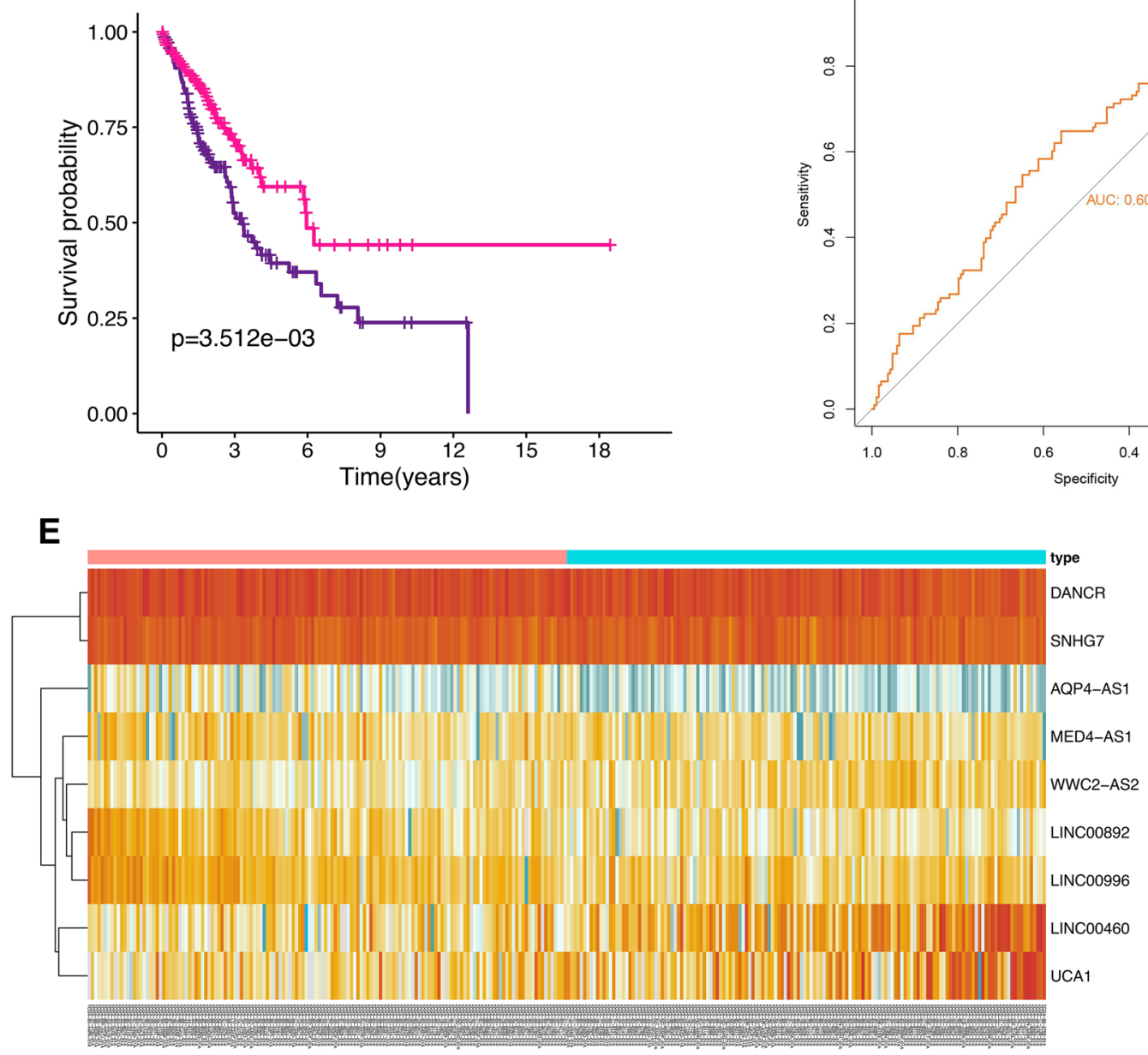

Fig. 7 Validation of the FRGs-IncRNAs signature. Distribution (A) and survival status plot (B) of NSCLC patients based on the median risk score. Kaplan-Meier survival (C) and ROC curve analysis (D) of the FRGs-IncRNAs signature in the internal testing group. (E) Heatmap of the expression profiles of the FRGs-IncRNAs in low- and high-risk groups. NSCLC, non-small cell lung cancer; IncRNAs: long non-coding RNAs; FRGs, ferroptosis-related genes; FRGs-IncRNAs: FRGs related IncRNAs; ROC, receiver operating characteristic 


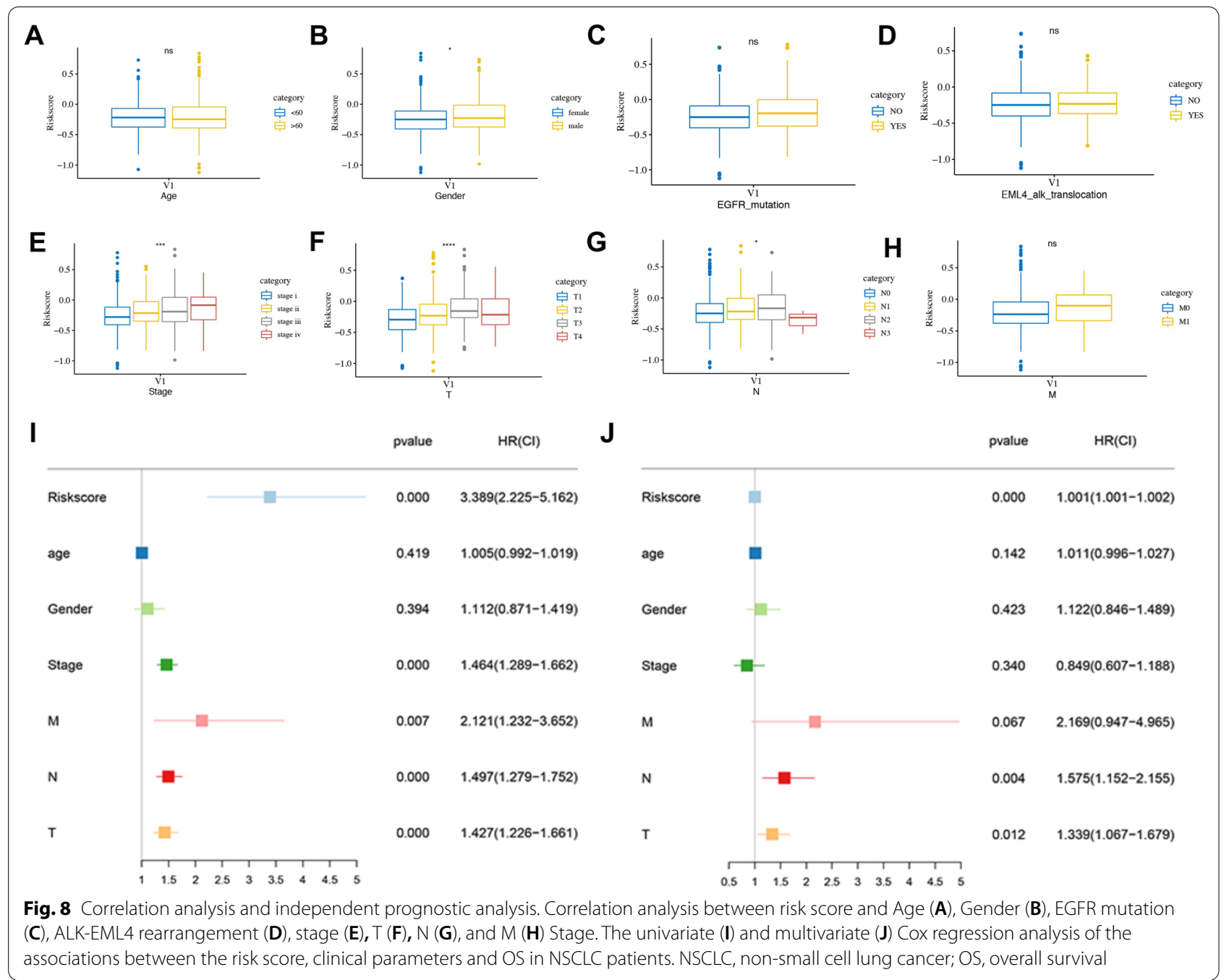

the previous study [48], MED4-AS1 is a protective factor for the prognosis of NSCLC in the present study, which is due to the down-regulated expression of MED4-AS1 in NSCLC compared with normal tissue.

Recently, many studies have confirmed that ferroptosis not only plays an important role in the occurrence and development of tumors but also affects tumor immunotherapy $[52,53]$. Induction of ferroptosis can enhance the effect of tumor immunotherapy. Hence, the expression of ICGs between the two risk groups was further investigated due to the crucial role of checkpoint inhibitor-based immunotherapies in NSCLC. We found different expression levels of BTLA, BTN2AA2, CD160, CD226, CD27, CD276, CD40LG, CD96, CTLA4, TIGIT between the two risk groups of patients with NSCLC. Of note, patients with high-risk scores were found highly sensitive to the chemotherapeutics cisplatin, docetaxel, erlotinib, and paclitaxel.
These results indicated that these FRGs-lncRNAs may regulate the development and progression of NSCLC by modulating the immune response and play a crucial role in the drug resistance in NSCLC.

The advantage of this study is that the data of LUAD and LUSC were systematically combined for the analysis and an FRGs-lncRNAs prognostic model of NSCLC was constructed for the first time and verified both in an internal and external cohort. What's more, the FRGs-lncRNAs were comprehensively identified both in the TCGA database and LncTarD database. Notably, the association between risk score and chemotherapeutics sensitivity was first analyzed in the present study. However, a prospective cohort and molecular biology experiment need to be conducted to further verify the accuracy of the prognostic model and the function of these FRGs-lncRNAs due to the lack of experimental verification in the present study. 


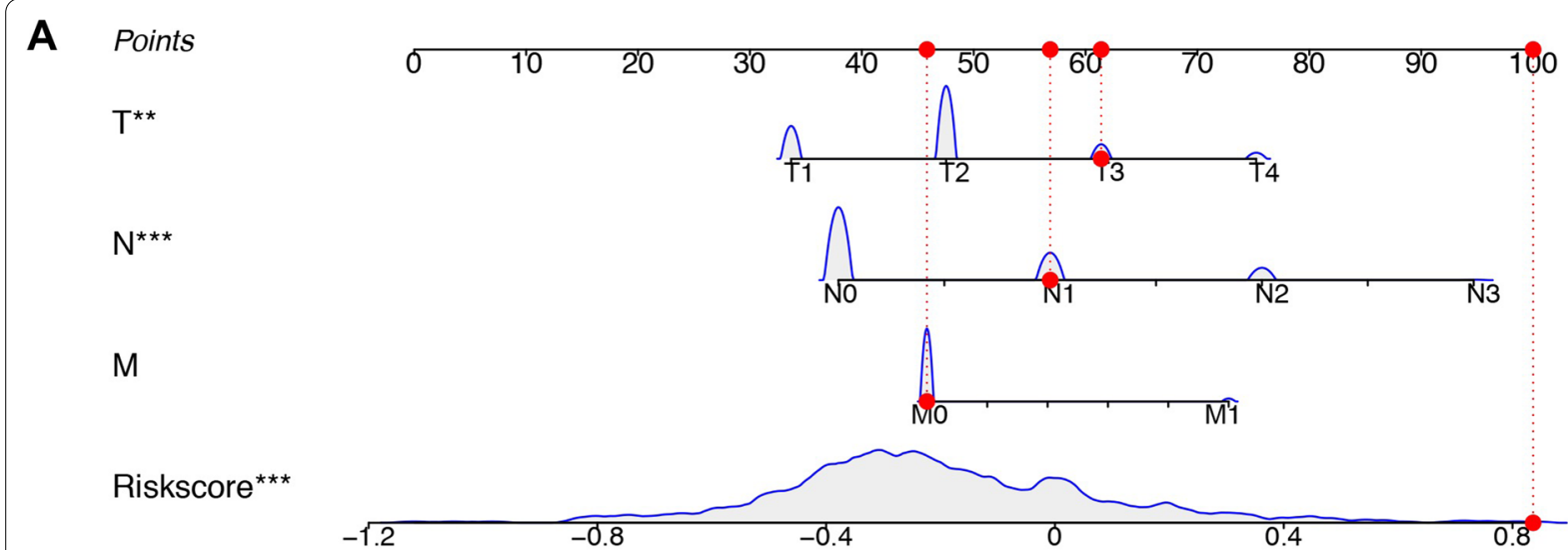

Total points

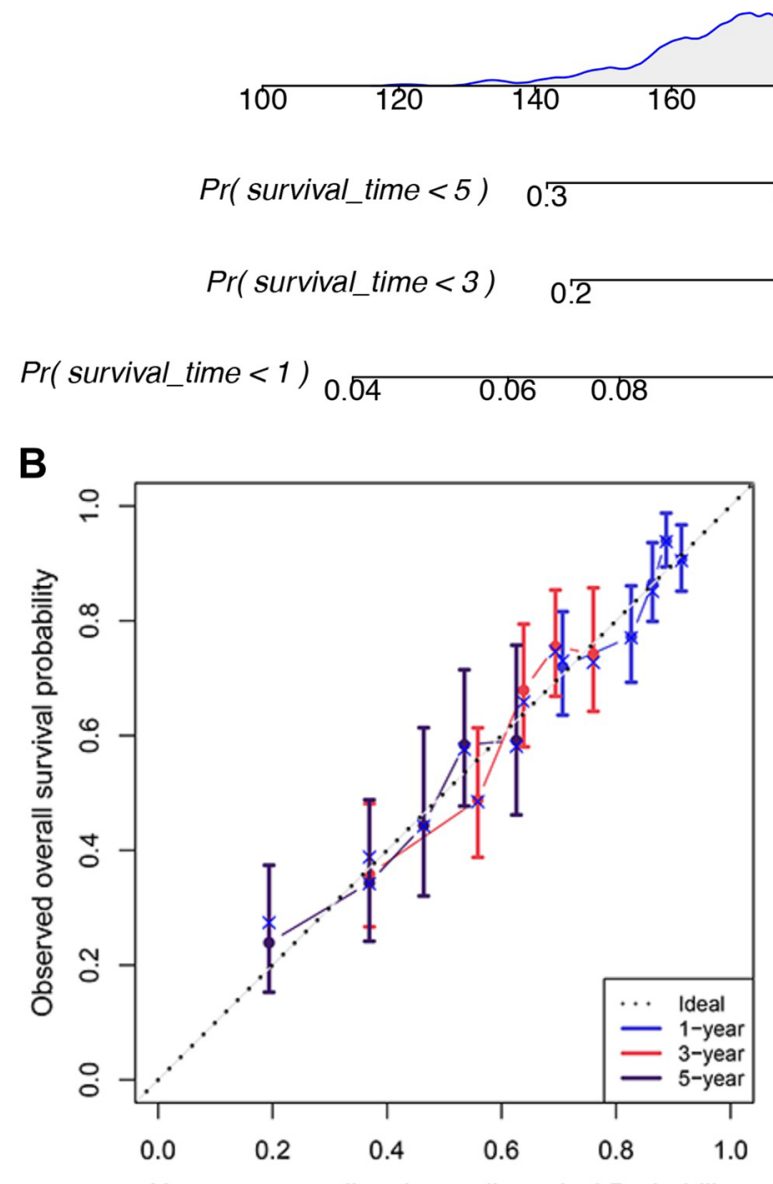

Nomogram predicted overall survival Probability

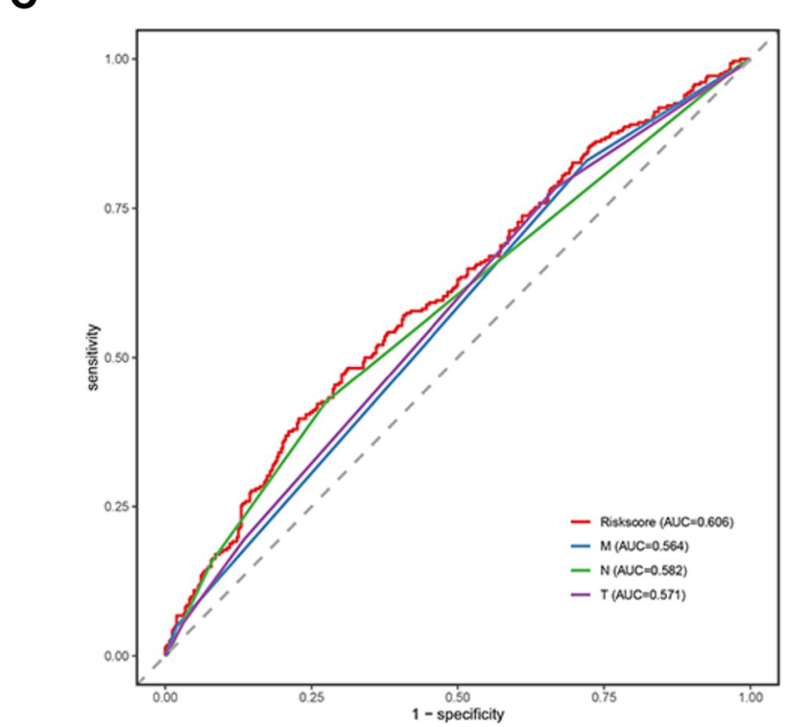

Fig. 9 Nomogram construction and evaluation. (A) The nomogram based on the clinic-pathological factors and risk score. (B) The calibration curves of the nomogram in predicting 1-, 3- and 5-years survival of NSCLC patients. (C) ROC curve of clinic-pathological factors and risk score for predicting prognosis of NSCLC. NSCLC, non-small cell lung cancer; OS, overall survival. FRGs, ferroptosis-related genes; FRGs-IncRNAs, FRGs-related IncRNAs; OS, overall survival

\section{Conclusion}

In conclusion, 10 FRGs-lncRNAs associated with the OS of NSCLC were identified and a novel
FRGs-lncRNAs prognostic model was constructed and verified both in the internal and external cohort in the present study. Then, the relationship between ICGs, 


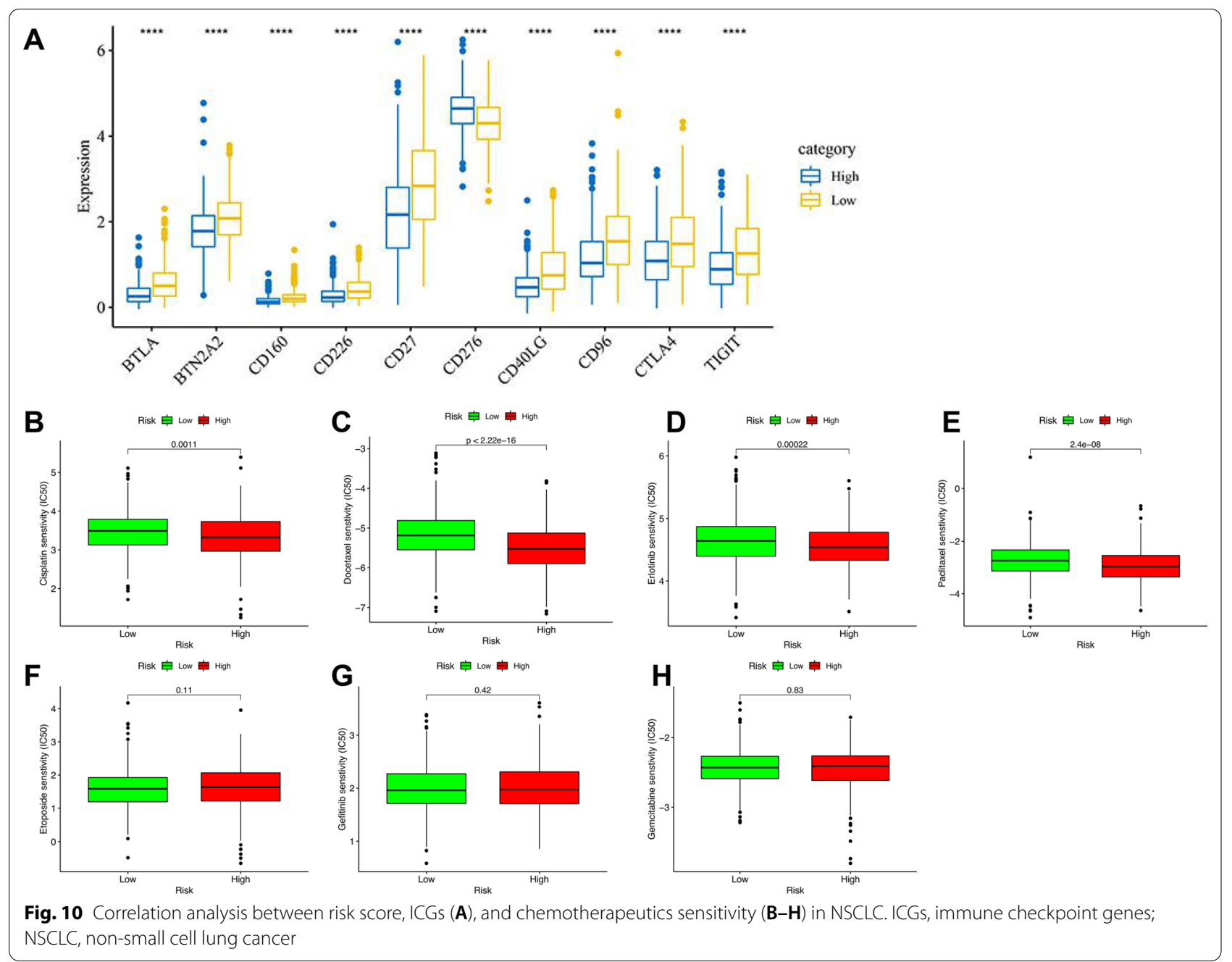

chemotherapeutics sensitivity and risk score were further evaluated. These findings have potential reference value for guiding the treatment and prognosis evaluation of NSCLC patients.

\section{Abbreviations}

NSCLC: Non-small cell lung cancer; FRGs: Ferroptosis-related genes; LUAD: Lung adenocarcinoma; OS: Overall survival; LUSC: Lung squamous cell carcinoma; TCGA: The Cancer Genome Atlas; DE-FRGs: Differentially expressed FRGs; FRGs-IncRNAs: FRGs-related IncRNAs; GO: Gene ontology; KEGG: Kyoto Encyclopedia of Genes and Genomes; K-M: Kaplan-Meier plotter; ROC: Receiver operational characteristic; ICGs: Immune checkpoint genes; AQP4-AS1: Aquaporin 4 antisense RNA 1; DANCR: Differentiation antagonizing non-protein-coding RNA; SNHG7: Small nucleolar RNA host gene 7; UCA1: Urothelial carcinoma-associated 1.

\section{Supplementary Information}

The online version contains supplementary material available at https://doi. org/10.1186/s12920-021-01133-4.

Additional file 1. Supplementary figures.
Additional file 2. Supplementary tables.

\section{Acknowledgements}

Not applicable.

\section{Authors' contributions}

Conception and design: PS, MC, ML; Administrative support: PS, MC; Data collection and assembly of data: ML, YZ, MF, HR; Data analysis and interpretation: ML, YZ, MF, HR; Data analysis and interpretation: All authors; Manuscript writing: All authors; Final approval of manuscript: All authors.

\section{Funding}

This work was funded by the National Natural Science Foundation of China (No. 81802291; to Puyu Shi); Key Research and Development Project of Science and Technology of Shaanxi Province (No. 2018KW-039, to Puyu Shi; No. 2021ZDLSF02-05, to Mingwei Chen; No. 2018SF-071, to Hui Ren); Fundamental Research Funds for the Central Universities in Xi'an Jiaotong University (No. 1191320149, to Puyu Shi; No. xzy012021064, to Hui Ren); China Postdoctoral Science Foundation Grant (No. 2019M653666, to Puyu Shi).

\section{Availability of data and materials}

The data of this study were obtained from the publicly available database (https://xena.ucsc.edu/). 


\section{Declarations}

\section{Ethics approval and consent to participate}

Ethics committee approval and informed consent of patients were not required in this study since all the expression, clinical information, and prognosis data of patients were obtained only from a public database. We are strictly in accordance with the declaration of Helsinki and the data access policies and publication guidelines of the TCGA database.

\section{Consent for publication}

Not applicable.

\section{Competing interests}

The authors declare that they have no competing interests.

\section{Author details}

'Department of Respiratory and Critical Care Medicine, The First Affiliated Hospital of Xi'an Jiao Tong University, No. 277, Yanta West Road, Xi'an 710061, Shaanxi, China. ${ }^{2}$ Department of Talent Highland, The First Affiliated Hospital of Xi'an Jiao Tong University, No. 277, Yanta West Road, Xi'an 710061, Shaanxi, China. ${ }^{3}$ Department of Thoracic Surgery, The First Affiliated Hospital of Xi'an Jiao Tong University, No. 277, Yanta West Road, Xi'an 710061, Shaanxi, China. ${ }^{4}$ Department of Center for Translational Medicine, The First Affiliated Hospital of Xi'an Jiao Tong University, No. 277, Yanta West Road, Xi'an 710061, Shaanxi, China.

Received: 4 August 2021 Accepted: 24 November 2021

Published online: 03 December 2021

\section{References}

1. Siegel RL, Miller KD, Jemal A. Cancer statistics, 2019. CA Cancer J Clin. 2019;69(1):7-34.

2. Cheng TY, Cramb SM, Baade PD, Youlden DR, Nwogu C, Reid ME. The international epidemiology of lung cancer: latest trends, disparities, and tumor characteristics. J Thorac Oncol. 2016;11(10):1653-71.

3. de Groot PM, Wu CC, Carter BW, Munden RF. The epidemiology of lung cancer. Transl Lung Cancer Res. 2018;7(3):220-33.

4. Qiao M, Jiang T, Liu X, et al. Immune checkpoint inhibitors in EGFRmutated non-small cell lung cancer: dusk or Dawn? J Thorac Oncol. 2021;16:1267-88

5. Doroshow DB, Sanmamed MF, Hastings K, et al. Immunotherapy in non-small cell lung cancer: facts and hopes. Clin Cancer Res. 2019;25(15):4592-602.

6. Ruiz-Cordero R, Devine WP. Targeted therapy and checkpoint immunotherapy in lung cancer. Surg Pathol Clin. 2020;13(1):17-33.

7. Tang D, Chen X, Kang R, Kroemer G. Ferroptosis: molecular mechanisms and health implications. Cell Res. 2021;31(2):107-25.

8. Stockwell BR, Friedmann Angeli JP, Bayir H, et al. Ferroptosis: a regulated cell death nexus linking metabolism, redox biology, and disease. Cell. 2017;171(2):273-85.

9. Xu G, Wang H, Li X, Huang R, Luo L. Recent progress on targeting ferroptosis for cancer therapy. Biochem Pharmacol. 2021;190:114584.

10. Shi X, Sun M, Liu H, Yao Y, Song Y. Long non-coding RNAs: a new frontier in the study of human diseases. Cancer Lett. 2013;339(2):159-66.

11. Chen Y, Zitello E, Guo R, Deng Y. The function of LncRNAs and their role in the prediction, diagnosis, and prognosis of lung cancer. Clin Transl Med. 2021;11(4):e367.

12. Jiang N, Zhang X, Gu X, Li X, Shang L. Progress in understanding the role of IncRNA in programmed cell death. Cell Death Discov. $2021 ; 7(1): 30$

13. Wang M, Mao C, Ouyang L, et al. Long noncoding RNA LINC00336 inhibits ferroptosis in lung cancer by functioning as a competing endogenous RNA. Cell Death Differ. 2019;26(11):2329-43.

14. Gai C, Liu C, Wu X, et al. MT1DP loaded by folate-modified liposomes sensitizes erastin-induced ferroptosis via regulating miR-365a-3p/NRF2 axis in non-small cell lung cancer cells. Cell Death Dis. 2020;11(9):751.
15. Mao C, Wang X, Liu Y, et al. A G3BP1-interacting IncRNA promotes ferroptosis and apoptosis in cancer via nuclear sequestration of p53. Cancer Res. 2018;78(13):3484-96.

16. Guo Y, Qu Z, Li D, et al. Identification of a prognostic ferroptosis-related IncRNA signature in the tumor microenvironment of lung adenocarcinoma. Cell Death Discov. 2021;7(1):190.

17. Tang Y, Li C, Zhang YJ, Wu ZH. Ferroptosis-related long non-coding RNA signature predicts the prognosis of head and neck squamous cell carcinoma. Int J Biol Sci. 2021;17(3):702-11.

18. Zhou R, Liang J, Tian H, Chen Q, Yang C, Liu C. Development of a ferroptosis-related IncRNA signature to predict the prognosis and immune landscape of bladder cancer. Dis Mark. 2021;2021:1031906.

19. Cai HJ, Zhuang ZC, Wu Y, et al. Development and validation of a ferroptosis-related IncRNAs prognosis signature in colon cancer. Bosn J Basic Med Sci. 2021;21(5):569-76.

20. Zhou N, Bao J. FerrDb: a manually curated resource for regulators and markers of ferroptosis and ferroptosis-disease associations. Database (Oxford). 2020. https://doi.org/10.1093/database/baaa021.

21. Hassannia B, Vandenabeele $P$, Vanden BT. Targeting ferroptosis to iron out cancer. Cancer Cell. 2019:35(6):830-49.

22. Liang JY, Wang DS, Lin HC, et al. A novel ferroptosis-related gene signature for overall survival prediction in patients with hepatocellular carcinoma. Int J Biol Sci. 2020;16(13):2430-41.

23. Bersuker K, Hendricks JM, Li Z, et al. The CoQ oxidoreductase FSP1 acts parallel to GPX4 to inhibit ferroptosis. Nature. 2019;575(7784):688-92.

24. Kanehisa M, Goto S. KEGG: kyoto encyclopedia of genes and genomes. Nucleic Acids Res. 2000;28(1):27-30.

25. Kanehisa M. Toward understanding the origin and evolution of cellular organisms. Protein Sci. 2019;28(11):1947-51.

26. Kanehisa M, Furumichi M, Sato Y, Ishiguro-Watanabe M, Tanabe M. KEGG: integrating viruses and cellular organisms. Nucleic Acids Res. 2021:49(D1):D545-d551.

27. Zhao H, Shi J, Zhang Y, et al. LncTarD: a manually-curated database of experimentally-supported functional IncRNA-target regulations in human diseases. Nucleic Acids Res. 2020;48(D1):D1 18-d126.

28. Tibshirani R. The lasso method for variable selection in the Cox model. Stat Med. 1997;16(4):385-95.

29. Simon N, Friedman J, Hastie T, Tibshirani R. Regularization paths for Cox's proportional hazards model via coordinate descent. J Stat Softw. 2011;39(5):1-13.

30. Hu FF, Liu CJ, Liu LL, Zhang Q, Guo AY. Expression profile of immune checkpoint genes and their roles in predicting immunotherapy response. Brief Bioinform. 2021. https://doi.org/10.1093/bib/bbaa176.

31. Molina JR, Yang P, Cassivi SD, Schild SE, Adjei AA. Non-small cell lung cancer: epidemiology, risk factors, treatment, and survivorship. Mayo Clin Proc. 2008;83(5):584-94.

32. Camidge DR, Doebele RC, Kerr KM. Comparing and contrasting predictive biomarkers for immunotherapy and targeted therapy of NSCLC. Nat Rev Clin Oncol. 2019;16(6):341-55

33. Herbst RS, Morgensztern D, Boshoff C. The biology and management of non-small cell lung cancer. Nature. 2018;553(7689):446-54.

34. Mou Y, Wang J, Wu J, et al. Ferroptosis, a new form of cell death: opportunities and challenges in cancer. J Hematol Oncol. 2019;12(1):34.

35. Guo J, Xu B, Han Q, et al. Ferroptosis: a novel anti-tumor action for cisplatin. Cancer Res Treat. 2018;50(2):445-60.

36. Yamaguchi Y, Kasukabe T, Kumakura S. Piperlongumine rapidly induces the death of human pancreatic cancer cells mainly through the induction of ferroptosis. Int J Oncol. 2018;52(3):1011-22.

37. Ma S, Henson ES, Chen Y, Gibson SB. Ferroptosis is induced following siramesine and lapatinib treatment of breast cancer cells. Cell Death Dis. 2016;7(7):e2307.

38. Sun X, Ou Z, Chen R, et al. Activation of the p62-Keap1-NRF2 pathway protects against ferroptosis in hepatocellular carcinoma cells. Hepatology. 2016;63(1):173-84.

39. Xie B, Guo Y. Molecular mechanism of cell ferroptosis and research progress in regulation of ferroptosis by noncoding RNAs in tumor cells. Cell Death Discov. 2021;7(1):101

40. Xing C, Cai Z, Gong J, Zhou J, Xu J, Guo F. Identification of potential biomarkers involved in gastric cancer through integrated analysis of noncoding RNA associated competing endogenous RNAs network. Clin Lab. 2018:64(10):1661-9. 
41. Marchi RD, Mathias C, Reiter GAK, et al. Association between SNP rs527616 in IncRNA AQP4-AS1 and susceptibility to breast cancer in a southern Brazilian population. Genet Mol Biol. 2021;44(1):e20200216.

42. Li K, Sun D, Gou Q, et al. Long non-coding RNA linc00460 promotes epithelial-mesenchymal transition and cell migration in lung cancer cells. Cancer Lett. 2018;420:80-90.

43. Nakano Y, Isobe K, Kobayashi H, et al. Clinical importance of long noncoding RNA LINC00460 expression in EGFR-mutant lung adenocarcinoma. Int J Oncol. 2020;56(1):243-57.

44. Chen X, Wang Z, Tong F, Dong X, Wu G, Zhang R. IncRNA UCA1 promotes gefitinib resistance as a ceRNA to target FOSL2 by sponging miR-143 in non-small cell lung cancer. Mol Ther Nucleic Acids. 2020;19:643-53.

45. Kretz M, Webster DE, Flockhart RJ, et al. Suppression of progenitor differentiation requires the long noncoding RNA ANCR. Genes Dev. 2012;26(4):338-43.

46. Jin SJ, Jin MZ, Xia BR, Jin WL. Long non-coding RNA DANCR as an emerging therapeutic target in human cancers. Front Oncol. 2019;9:1225.

47. Bai Y, Zhang G, Chu H, Li P, Li J. The positive feedback loop of IncRNA DANCR/miR-138/Sox4 facilitates malignancy in non-small cell lung cancer. Am J Cancer Res. 2019;9(2):270-84.

48. Wang XW, Guo QQ, Wei Y, et al. Construction of a competing endogenous RNA network using differentially expressed IncRNAs, miRNAs and mRNAs in non-small cell lung cancer. Oncol Rep. 2019;42(6):2402-15.

49. Pei YF, He Y, Hu LZ, Zhou B, Xu HY, Liu XQ. The crosstalk between IncRNASNHG7/miRNA-181/cbx7 modulates malignant character in lung adenocarcinoma. Am J Pathol. 2020;190(6):1343-54.

50. Shan Y, Ma J, Pan Y, Hu J, Liu B, Jia L. LncRNA SNHG7 sponges miR-216b to promote proliferation and liver metastasis of colorectal cancer through upregulating GALNT1. Cell Death Dis. 2018;9(7):722.

51. Chen K, Abuduwufuer A, Zhang H, Luo L, Suotesiyali M, Zou Y. SNHG7 mediates cisplatin-resistance in non-small cell lung cancer by activating PI3K/AKT pathway. Eur Rev Med Pharmacol Sci. 2019;23(16):6935-43.

52. Wang W, Green M, Choi JE, et al. CD8(+) T cells regulate tumour ferroptosis during cancer immunotherapy. Nature. 2019;569(7755):270-4.

53. Stockwell BR, Jiang X. A physiological function for ferroptosis in tumor suppression by the immune system. Cell Metab. 2019;30(1):14-5.

\section{Publisher's Note}

Springer Nature remains neutral with regard to jurisdictional claims in published maps and institutional affiliations.

Ready to submit your research? Choose BMC and benefit from:

- fast, convenient online submission

- thorough peer review by experienced researchers in your field

- rapid publication on acceptance

- support for research data, including large and complex data types

- gold Open Access which fosters wider collaboration and increased citations

- maximum visibility for your research: over $100 \mathrm{M}$ website views per year

At BMC, research is always in progress.

Learn more biomedcentral.com/submissions 